Research Article

\title{
Investigation of Pseudo-Ricci Symmetric Spacetimes in Gray's Subspaces
}

\author{
Sameh Shenawy, ${ }^{1}$ Uday Chand De, ${ }^{2}$ Nasser Bin Turki, ${ }^{3}$ Suliman Alsaeed, ${ }^{4}$ \\ and Abdallah Abdelhameed Syied $\mathbb{1}^{5}$ \\ ${ }^{1}$ Basic Science Department, Modern Academy for Engineering and Technology, Maadi, Egypt \\ ${ }^{2}$ Department of Pure Mathematics, University of Calcutta, 35, Ballygaunge Circular Road, Kolkata 700019, West Bengal, India \\ ${ }^{3}$ Department of Mathematics, College of Science, King Saud University, P.O. Box 2455, Riyadh 11451, Saudi Arabia \\ ${ }^{4}$ Applied Science College, Department of Mathematical Sciences, Umm Al-Qura University, P.O. Box 715, Makkah 21955, \\ Saudi Arabia \\ ${ }^{5}$ Department of Mathematics, Faculty of Science, Zagazig University, P.O. Box 44519, Zagazig, Egypt
}

Correspondence should be addressed to Abdallah Abdelhameed Syied; a.a_syied@yahoo.com

Received 5 July 2021; Accepted 9 August 2021; Published 17 August 2021

Academic Editor: Bibhas Ranjan Majhi

Copyright (c) 2021 Sameh Shenawy et al. This is an open access article distributed under the Creative Commons Attribution License, which permits unrestricted use, distribution, and reproduction in any medium, provided the original work is properly cited.

In the present paper, we focused our attention to study pseudo-Ricci symmetric spacetimes in Gray's decomposition subspaces. It is proved that (PRS) ${ }_{n}$ spacetimes are Ricci flat in trivial, $A$, and $B$ subspaces, whereas perfect fluid in subspaces $I, I \oplus A$, and $I \oplus B$, and have zero scalar curvature in subspace $A \oplus B$. Finally, it is proved that pseudo-Ricci symmetric GRW spacetimes are vacuum, and as a consequence of this result, we address several corollaries.

\section{Introduction}

A pseudo-Ricci symmetric manifold (briefly $(\mathrm{PRS})_{n}$ ) is a nonflat pseudo-Riemannian manifold whose Ricci tensor satisfies

$$
\nabla_{k} R_{i j}=2 A_{k} R_{i j}+A_{i} R_{k j}+A_{j} R_{i k}
$$

where $A$ is a nonzero 1 -form and $\nabla$ indicates the covariant differentiation with respect to the metric $g$ [1].

The class of pseudo-Ricci symmetric manifolds is a subclass of weakly Ricci symmetric manifolds which were first introduced and studied by Tamássy and Binh [2]. There has been much focus on the concept of (PRS $)_{n}$ manifolds; for instance, a sufficient condition on (PRS $)_{n}$ manifolds to be quasi-Einstein manifolds was introduced by De and Gazi [3]. (PRS) ${ }_{n}$ manifolds whose scalar curvature satisfies $\nabla_{k} R=$ 0 have zero scalar curvature [1]. A concrete example of pseudo-Ricci symmetric manifolds was given in [4]. There are many generalizations of (PRS $)_{n}$ manifolds, for example, see $[5,6]$.

An invariant orthogonal decomposition of the covariant derivative of the Ricci tensor was coined and studied by Gray in [7] (see also [8-10]). The manifolds in the trivial subspace have parallel Ricci tensor; that is, $\nabla_{k} R_{i j}=0$. The subspace $\mathscr{A}$ contains manifolds whose Ricci tensor is Killing; that is,

$$
\nabla_{j} R_{k i}+\nabla_{k} R_{j i}+\nabla_{i} R_{k j}=0 .
$$

The next subspace is denoted by $\mathscr{B}$. The Ricci tensors of manifolds in $\mathscr{B}$ are Codazzi; that is,

$$
\nabla_{k} R_{i j}=\nabla_{i} R_{k j}
$$

The subspace $\mathscr{A} \oplus \mathscr{B}$ is characterized by the equation $\nabla R=0$. Manifolds with

$$
\nabla_{k} R_{i j}=\frac{n}{(n-1)(n+2)} \nabla\left(k R g_{i j}\right)
$$


lie in $\mathscr{I}$. In $\mathscr{I} \oplus \mathscr{A}$, the tensor $R_{i j}-(2 R /(n+2)) g_{i j}$ is Killing, whereas in $\mathscr{I} \oplus \mathscr{B}$, the tensor $R_{j i}-(R / 2(n-1)) g_{j i}$ is a Codazzi tensor. Such manifolds are called Einstein-like manifolds [11]. Recently, there has been growing interest in this decomposition. For example, generalized Robertson-Walker spacetimes are either Einstein or perfect fluid in Gray's orthogonal subspaces except one in which the Ricci tensor is not restricted [12].

An $n$-dimensional Lorentzian manifold is said to be pseudo-Ricci symmetric spacetime if the Ricci tensor satisfies equation (1). Here, we assume the associated vector $A_{i}$ is a unit time-like vector $\left(A_{i} A^{i}=-1\right)$.

In standard theory of gravity, the relation between the matter of spacetimes and the geometry of spacetimes is given by Einstein's field equation (EFE):

$$
R_{i j}-\frac{R}{2} g_{i j}=k T_{i j}
$$

where $R_{i j}, R, k$, and $T_{i j}$ are the Ricci tensor, scalar curvature tensor, Newtonian constant, and energy-momentum tensor, respectively. EFE implies that the energy-momentum tensor $T_{i j}$ is divergence-free. This requirement is directly satisfied if $\nabla_{l} T_{i j}=0$.

This paper is organized as follows: In Section 2, general properties of $(\mathrm{PRS})_{n}$ spacetimes are considered. In Section 3, $(\mathrm{PRS})_{n}$ spacetimes are investigated in all Gray's orthogonal subspaces. It is proved that (PRS) ${ }_{n}$ spacetimes in trivial, $\mathscr{A}$, and $\mathscr{B}$ subspaces are Ricci flat, in subspaces $\mathscr{I}, \mathscr{I} \oplus \mathscr{A}$, and $\mathscr{I} \oplus \mathscr{B}$ are perfect fluid spacetimes, and in $\mathscr{A} \oplus \mathscr{B}$ have a zero scalar curvature. In Section 4, we prove that pseudo-Ricci symmetric GRW spacetimes are vacuum and as a consequence, we address some corollaries.

\section{On (PRS $)_{n}$ Spacetimes}

In this section, the main properties of $(\mathrm{PRS})_{n}$ spacetimes are considered. Equation (1) implies

$$
\nabla_{k} R_{i}^{k}=3 A^{j} R_{i j}+A_{i} R
$$

The use of $\nabla_{k} R_{i}^{k}=(1 / 2) \nabla_{i} R$ yields

$$
\nabla_{i} R=6 A^{j} R_{i j}+2 A_{i} R \text {. }
$$

A different contraction of equation (1) with $g^{i j}$ gives

$$
\nabla_{k} R=2 A_{k} R+2 A^{j} R_{k j}
$$

Solving equations (7) and (8) together, one gets

$$
\begin{aligned}
A^{j} R_{k j} & =0, \\
\nabla_{k} R & =2 A_{k} R .
\end{aligned}
$$

Lemma 1. In $(P R S)_{n}$ spacetimes, the covariant derivative of the scalar curvature is $\nabla_{k} R=2 A_{k} R$. Moreover, $A^{j}$ is an eigenvector of the Ricci tensor $R_{i j}$ with zero eigenvalue.

Assume that the scalar curvature is constant. Equation (10) directly leads to $R=0$.
Lemma 2. In (PRS) spacetimes, the scalar curvature $R$ is constant if and only if $R=0$.

Let us consider $R \neq 0$; then, the use of equation (10) in equation (1) implies that

$$
\nabla_{k} R_{i j}=\frac{\nabla_{k} R}{R} R_{i j}+\frac{\nabla_{i} R}{2 R} R_{k j}+\frac{\nabla_{j} R}{2 R} R_{i k} .
$$

This leads us to the following lemma.

Lemma 3. In $(P R S)_{n}$ spacetimes with nonzero scalar curvature, the covariant derivative of the Ricci tensor takes the form

$$
\nabla_{k} R_{i j}=\frac{\nabla_{k} R}{R} R_{i j}+\frac{\nabla_{i} R}{2 R} R_{k j}+\frac{\nabla_{j} R}{2 R} R_{i k},
$$

provided $R \neq 0$.

The Weyl tensor of type $(0,4)$ has the form [13]

$$
\begin{aligned}
C_{i j k l}= & R_{i j k l}-\frac{1}{n-1}\left\{g_{i l} R_{j k}+g_{j k} R_{i l}-g_{i k} R_{j l}-g_{j l} R_{i k}\right\} \\
& +\frac{R}{(n-1)(n-2)}\left\{g_{i l} g_{j k}-g_{i k} g_{j l}\right\},
\end{aligned}
$$

and its divergence is

$$
\nabla_{h} \mathscr{C}_{i j k}^{h}=\frac{n-3}{n-2}\left[\left(\nabla_{k} R_{i j}-\nabla_{j} R_{i k}\right)-\frac{1}{2(n-1)}\left(g_{i j} \nabla_{k} R-g_{i k} \nabla_{j} R\right)\right] .
$$

In virtue of (1) and (10), we have

$$
\nabla_{h} \mathscr{C}_{i j k}^{h}=\frac{n-3}{n-2}\left[\left(A_{k} R_{i j}-A_{j} R_{i k}\right)-\frac{1}{(n-1)}\left(g_{i j} A_{k} R-g_{i k} A_{j} R\right)\right] \text {. }
$$

Assume that the Weyl conformal curvature tensor is divergence-free, that is, $\nabla_{h} \mathscr{C}_{i j k}^{h}=0$; then,

$$
A_{k} R_{i j}-A_{j} R_{i k}=\frac{(n-2)}{(n-1)(n-3)}\left(g_{i j} A_{k} R-g_{i k} A_{j} R\right) .
$$

Contracting with $A^{k}$ and using equation (9), we obtain

$$
R_{i j}=\frac{(n-2) R}{(n-1)(n-3)}\left(g_{i j}+A_{i} A_{j}\right) \text {. }
$$

A multiplication with $g^{i j}$ gives $R=0$, and hence,

$$
R_{i j}=0 \text {. }
$$

Thus, we can conclude the following theorem:

Theorem 1. A $(P R S)_{n}$ spacetime with divergence-free Weyl curvature tensor is Ricci flat.

The use of this result $\left(R_{i j}=0\right)$ in the defining property of the conformal curvature tensor entails that

$$
\mathscr{C}_{i j k h}=R_{i j k h} \text {. }
$$

Hence, we have the following corollary. 
Corollary 1. Semisymmetric and conformally semisymmetric pseudo-Ricci symmetric spacetimes are equivalent.

The covariant derivative of equation (1) gives

$$
\nabla_{r} \nabla_{k} R_{i j}=2 \nabla_{r}\left(A_{k} R_{i j}\right)+\nabla_{r}\left(A_{i} R_{k j}\right)+\nabla_{r}\left(A_{j} R_{i k}\right) \text {. }
$$
have

Interchanging the indices $r$ and $k$ in the last equation, we

$$
\nabla_{k} \nabla_{r} R_{i j}=2 \nabla_{k}\left(A_{r} R_{i j}\right)+\nabla_{k}\left(A_{i} R_{r j}\right)+\nabla_{k}\left(A_{j} R_{i r}\right) .
$$

Subtracting the last two equations, we obtain

$$
\begin{aligned}
\left(\nabla_{r} \nabla_{k}-\nabla_{k} \nabla_{r}\right) R_{i j}= & 2 \nabla_{r}\left(A_{k} R_{i j}\right)+\nabla_{r}\left(A_{i} R_{k j}\right)+\nabla_{r}\left(A_{j} R_{i k}\right) \\
& -2 \nabla_{k}\left(A_{r} R_{i j}\right)-\nabla_{k}\left(A_{i} R_{r j}\right)-\nabla_{k}\left(A_{j} R_{i r}\right), \\
\left(\nabla_{r} \nabla_{k}-\nabla_{k} \nabla_{r}\right) R_{i j}= & 2 R_{i j} \nabla_{r}\left(A_{k}\right)+R_{k j} \nabla_{r}\left(A_{i}\right)+R_{i k} \nabla_{r}\left(A_{j}\right) \\
& +2 A_{k} \nabla_{r}\left(R_{i j}\right)+A_{i} \nabla_{r}\left(R_{k j}\right)+A_{j} \nabla_{r}\left(R_{i k}\right) \\
& -2 A_{r} \nabla_{k}\left(R_{i j}\right)-A_{i} \nabla_{k}\left(R_{r j}\right)-A_{j} \nabla_{k}\left(R_{i r}\right) \\
& -2 R_{i j} \nabla_{k}\left(A_{r}\right)-R_{r j} \nabla_{k}\left(A_{i}\right)-R_{i r} \nabla_{k}\left(A_{j}\right) .
\end{aligned}
$$

Making use of equation (1) and simplifying, we get

$$
\begin{aligned}
\left(\nabla_{r} \nabla_{k}-\nabla_{k} \nabla_{r}\right) R_{i j}= & 2 R_{i j}\left[\nabla_{r}\left(A_{k}\right)-\nabla_{k}\left(A_{r}\right)\right]+R_{k j} \nabla_{r}\left(A_{i}\right) \\
& +R_{i k} \nabla_{r}\left(A_{j}\right)-R_{r j} \nabla_{k}\left(A_{i}\right)-R_{i r} \nabla_{k}\left(A_{j}\right) \\
& +A_{i} A_{k} R_{j r}+A_{i} A_{j} R_{r k}+A_{j} A_{k} R_{i r} \\
& -A_{i} A_{r} R_{k j}-A_{j} A_{r} R_{k i} .
\end{aligned}
$$

Now, assume that the (PRS) $)_{n}$ is Ricci semisymmetric, that is, $\left(\nabla_{r} \nabla_{k}-\nabla_{k} \nabla_{r}\right) R_{i j}=0$; we have

$$
\begin{aligned}
0= & 2 R_{i j}\left[\nabla_{r}\left(A_{k}\right)-\nabla_{k}\left(A_{r}\right)\right]+R_{k j} \nabla_{r}\left(A_{i}\right) \\
& +R_{i k} \nabla_{r}\left(A_{j}\right)-R_{r j} \nabla_{k}\left(A_{i}\right)-R_{i r} \nabla_{k}\left(A_{j}\right) \\
& +A_{i} A_{k} R_{j r}+A_{i} A_{j} R_{r k}+A_{j} A_{k} R_{i r}-A_{i} A_{r} R_{k j}-A_{j} A_{r} R_{k i} .
\end{aligned}
$$

Contracting with $A^{j}$ and using equation (9), we infer

$$
-A_{i} R_{r k}+A_{r} R_{k i}=0 .
$$

Again, contracting with $A^{i}$ and utilizing equation (9), we get

$$
R_{r k}=0 .
$$

Thus, we have the following theorem:

Theorem 2. Ricci semisymmetric $(P R S)_{n}$ spacetimes are Ricci flat.

\section{ERROR!!PRS) ${ }_{n}$ Spacetimes in Gray's Decomposition Subspaces}

This section is devoted to study (PRS) ${ }_{n}$ spacetimes in Gray's seven subspaces. Three main results are obtained in this section. A Lorentzian manifold $M$ is said to be perfect fluid if its Ricci tensor satisfies

$$
R_{i j}=\alpha g_{i j}+\beta u_{i} u_{j}
$$

where $\alpha$ and $\beta$ are scalar fields and $u_{i}$ is a time-like vector field [14].

Theorem 3. $(P R S)_{n}$ spacetimes in trivial, $\mathscr{A}$, and $\mathscr{B}$ subspaces are Ricci flat.

Proof. The trivial subspace of Gray's decomposition contains spacetimes whose Ricci tensors are parallel and the scalar curvatures are constant. Thus, equation (10) easily gives $R=0$. And hence, equation (1) becomes

$$
2 A_{k} R_{i j}=-A_{i} R_{k j}-A_{j} R_{i k}
$$

A contraction of equation (28) with $g^{i j}$ yields

$$
R_{i j}=R A_{i} A_{j}=0 .
$$

And consequently,

$$
R_{i j}=0 \text {, }
$$

which means that $(\mathrm{PRS})_{n}$ spacetimes with parallel Ricci tensor are Ricci flat.

In subspace $\mathscr{A}(\mathrm{PRS})_{n}$ spacetimes have a Killing Ricci tensor; that is,

$$
\nabla_{j} R_{k i}+\nabla_{k} R_{j i}+\nabla_{i} R_{k j}=0 .
$$

It is well known that in this subspace, the scalar curvature is covariantly constant. Equation (10) implies $R=0$. Using equation (1) in equation (31), we have

$$
A_{k} R_{i j}+A_{j} R_{i k}+A_{i} R_{j k}=0 .
$$

Contracting equation (32) with $A^{k}$ and using equation (9), we get

$$
R_{i j}=R A_{j} A_{i}=0,
$$

which means that $(\mathrm{PRS})_{n}$ spacetimes in subspace $\mathscr{A}$ are Ricci flat.

Next, let us consider the subspace $\mathscr{B}$ in which $(\mathrm{PRS})_{n}$ has a Codazzi type of Ricci tensor [15]. The Codazzi deviation tensor $D_{i j k}$ of $(\mathrm{PRS})_{n}$ is given by

$$
\begin{aligned}
D_{i j k} & =\nabla_{k} R_{i j}-\nabla_{i} R_{k j} \\
& =2 A_{k} R_{i j}+A_{i} R_{k j}+A_{j} R_{i k}-\left[2 A_{i} R_{k j}+A_{k} R_{i j}+A_{j} R_{i k}\right] \\
& =A_{k} R_{i j}-A_{i} R_{k j} .
\end{aligned}
$$

A contraction with $g^{i j}$ implies

$$
g^{i j} D_{i j k}=A_{k} R-A^{j} R_{k j} .
$$

But, in this subspace, the spacetimes have Codazzi-type Ricci tensor (that is, $D_{i j k}=0$ ); then,

$$
A_{k} R_{i j}=A_{i} R_{k j}
$$


Multiplying with $g^{i j}$ and utilizing equation (9), we get

$$
R=0 \text {. }
$$

A contraction of equation (36) by $A^{k}$ gives

$$
R_{i j}=0 \text {, }
$$

which means that $(\mathrm{PRS})_{n}$ spacetimes in Gray's subspace $\mathscr{B}$ are Ricci flat.

Theorem 4. $(P R S)_{n}$ spacetimes in $\mathscr{I}, \mathscr{I} \oplus \mathscr{A}$, and $\mathscr{I} \oplus \mathscr{B}$ subspaces are perfect fluid spacetimes.

Proof. In subspace $\mathscr{I}$, the Ricci tensor of pseudo-Ricci symmetric manifold $M$ satisfies the following property:

$$
\begin{aligned}
\nabla_{k} R_{i j}= & \frac{n \nabla_{k} R}{(n-1)(n+2)} g_{i j}+\frac{(n-2) \nabla_{i} R}{2(n-1)(n+2)} g_{k j} \\
& +\frac{(n-2) \nabla_{j} R}{2(n-1)(n+2)} g_{i k} .
\end{aligned}
$$

Applying equation (1), we obtain

$$
\begin{aligned}
2 A_{k} R_{i j}+A_{i} R_{k j}+A_{j} R_{i k}= & \frac{n \nabla_{k} R}{(n-1)(n+2) g_{i j}}+\frac{(n-2) \nabla_{i} R}{2(n-1)(n+2)} g_{k j} \\
& +\frac{(n-2) \nabla_{j} R}{2(n-1)(n+2)} g_{i k} .
\end{aligned}
$$

It follows that

$$
\begin{aligned}
2 A_{k} R_{i j}+A_{i} R_{k j}+A_{j} R_{i k}= & \frac{2 n A_{k} R}{(n-1)(n+2) g_{i j}}+\frac{(n-2) A_{i} R}{(n-1)(n+2)} g_{k j} \\
& +\frac{(n-2) A_{j} R}{(n-1)(n+2)} g_{i k} .
\end{aligned}
$$

Contracting with $A^{k}$ implies

$$
R_{i j}=\frac{n R}{(n-1)(n+2)} g_{i j}+\frac{(2-n) R}{(n-1)(n+2)} A_{i} A_{j},
$$

which means that $(\mathrm{PRS})_{n}$ spacetimes in subspace $\mathscr{I}$ are perfect fluid.

In subspace $\mathscr{I} \oplus \mathscr{A}$, the Ricci curvature tensor satisfies

$$
\nabla_{k} R_{i j}+\nabla_{i} R_{k j}+\nabla_{j} R_{i k}=\frac{2 \nabla_{k} R}{(n+2)} g_{i j}+\frac{2 \nabla_{i} R}{(n+2)} g_{k j}+\frac{2 \nabla_{j} R}{(n+2)} g_{i k} \text {. }
$$

Using equation (1), we infer

$$
A_{k} R_{i j}+A_{j} R_{i k}+A_{i} R_{j k}=\frac{2}{(n+2)}\left(\nabla_{k} R g_{i j}+\nabla_{i} R g_{k j}+\nabla_{j} R g_{i k}\right) \text {. }
$$

Now, equation (10) implies

$$
A_{k} R_{i j}+A_{j} R_{i k}+A_{i} R_{j k}=\frac{2 R}{(n+2)}\left(A_{k} g_{i j}+A_{i} g_{k j}+A_{j} g_{i k}\right) .
$$

A contraction with $A^{k}$ yields

$$
R_{i j}=\frac{2 R}{(n+2)}\left(g_{i j}-2 A_{j} A_{i}\right)
$$

which means that $(\mathrm{PRS})_{n}$ spacetimes in subspace $\mathscr{I} \oplus \mathscr{A}$ are perfect fluid.

Assume that $(\mathrm{PRS})_{n}$ are in Gray's subspace $\mathscr{I} \oplus \mathscr{B}$; that is,

$$
\nabla_{k} R_{j i}-\nabla_{j} R_{k i}=\frac{1}{2(n-1)}\left[g_{j i} \nabla_{k} R-g_{k i} \nabla_{j} R\right] .
$$

Equation (1) implies

$$
2(n-1)\left(A_{k} R_{i j}-A_{j} R_{k i}\right)=g_{j i} \nabla_{k} R-g_{k i} \nabla_{j} R .
$$

The use of equation (10) gives

$$
(n-1)\left(A_{k} R_{i j}-A_{j} R_{k i}\right)=g_{j i} A_{k} R-g_{k i} A_{j} R .
$$

Contracting with $A^{k}$, we obtain

$$
R_{i j}=\frac{R}{n-1}\left(g_{j i}+A_{i} A_{j}\right)
$$

which means that (PRS) ${ }_{n}$ spacetimes in Gray's subspace $\mathscr{I} \oplus \mathscr{B}$ are perfect fluid.

Theorem 5. $(P R S)_{n}$ spacetimes in $\mathscr{A} \oplus \mathscr{B}$ subspace have zero scalar curvature.

Proof. In subspace $\mathscr{A} \oplus \mathscr{B}$, the scalar curvature is covariantly constant and hence equation (10) implies

$$
R=0 \text {, }
$$

which means $(\mathrm{PRS})_{n}$ spacetimes in Gray's subspace $\mathscr{A} \oplus \mathscr{B}$ have zero scalar curvature.

\section{Pseudo-Ricci Symmetric GRW Spacetimes}

A generalized Robertson-Walker spacetime (for simplicity, denoted by GRW spacetimes) is the warped product $M=$ $I \times{ }_{f} M^{*}$ of an open connected interval $\left(I,-\mathrm{d} t^{2}\right)$ and a Riemannian manifold $M^{*}$, where $f: I \longrightarrow \mathbb{R}^{+}$is a positive smooth function. A Lorentzian manifold $M$ is a generalized Robertson-Walker spacetime if and only if $M$ possesses a unit time-like vector field $u_{i}$ with $[16,17]$

$$
\begin{aligned}
& \nabla_{k} u_{i}=\varphi\left(g_{k i}+u_{k} u_{i}\right), \\
& R_{i j} u^{j}=\xi u_{i},
\end{aligned}
$$

where $\varphi$ and $\xi$ are scalar functions. Vector fields satisfying equation (52) are called torse-forming. 
Now, assume that $M$ is a (PRS) ${ }_{n}$ generalized Robertson-Walker spacetime; that is,

$$
\nabla_{k} R_{i j}=2 A_{k} R_{i j}+A_{i} R_{k j}+A_{j} R_{i k} .
$$

A contraction with $u^{j}$ yields

$$
u^{j} \nabla_{k} R_{i j}=2 A_{k}\left(u^{j} R_{i j}\right)+A_{i}\left(u^{j} R_{k j}\right)+A_{j}\left(u^{j} R_{i k}\right) .
$$

Using equation (1), one gets

$$
u^{j} \nabla_{k} R_{i j}=2 A_{k} \xi u_{i}+\xi A_{i} u_{k}+u^{j} A_{j} R_{i k} .
$$

Therefore,

$$
u^{j} \nabla_{k} R_{i j}=2 \xi A_{k} u_{i}+\xi A_{i} u_{k}+\left(u^{j} A_{j}\right) R_{i k} .
$$

However,

$$
\begin{aligned}
u^{j} \nabla_{k} R_{i j} & =\nabla_{k}\left(R_{i j} u^{j}\right)-R_{i j} \nabla_{k} u^{j} \\
& =u_{i} \nabla_{k} \xi+\xi \nabla_{k}\left(u_{i}\right)-R_{i j} \nabla_{k} u^{j} \\
& =u_{i} \nabla_{k} \xi+\varphi\left(\xi g_{k i}-R_{i k}\right) .
\end{aligned}
$$

Thus,

$$
u_{i} \nabla_{k} \xi+\varphi\left(\xi g_{k i}-R_{i k}\right)=2 \xi A_{k} u_{i}+\xi A_{i} u_{k}+\left(u^{j} A_{j}\right) R_{i k} .
$$

It is well known $\nabla_{k} \xi=-u_{k}\left(u^{j} \nabla_{j} \xi\right)=-\rho u_{k}$, where $\rho=$ $\left(u^{j} \nabla_{j} \xi\right)$ (see [12]); thus,

$\left(\varphi+u^{j} A_{j}\right) R_{i k}=\xi \varphi g_{k i}-\rho u_{i} u_{k}-2 \xi A_{k} u_{i}-\xi A_{i} u_{k}$.

Since $M$ is (PRS) ${ }_{n}$, equation (9) shows that

$$
A^{j} R_{k j}=0 .
$$

Multiplying both the sides by $u^{k}$, that is,

$$
A^{j}\left(R_{k j} u^{k}\right)=0 .
$$

Using equation (53), one gets

$$
\xi A^{j} u_{j}=0 .
$$

Now, there are two different possible cases. The first one $A^{j} u_{j}=0$ and consequently $\xi$ does not vanish. Then, equation (59) becomes

$$
\varphi R_{i k}=\xi \varphi g_{k i}-\rho u_{i} u_{k}-2 \xi A_{k} u_{i}-\xi A_{i} u_{k} .
$$

A contraction by $A^{i}$ implies that

$$
0=\xi \varphi A_{k}-\xi\left(A^{i} A_{i}\right) u_{k},
$$

which is a contradiction. The second case is $\xi=0$. Then, equation (60) leads to

$$
\left(\varphi+u^{j} A_{j}\right) R_{i k}=0 .
$$

Thus, either $R_{i k}=0$ or $\varphi=-u^{j} A_{j}$.

Theorem 6. A pseudo-Ricci symmetric GRW spacetime is vacuum provided the one form $A$ is not codirectional with the torse-forming vector field $u$.
Suppose $A_{i} \neq \varphi u_{i}$. Then, the spacetime under consideration is Ricci flat, that is, $R_{i j}=0$, which implies $R=0$. It is known that

$$
\nabla_{h} \mathscr{C}_{i j k}^{h}=\frac{n-3}{n-2}\left[\left(\nabla_{k} R_{i j}-\nabla_{j} R_{i k}\right)-\frac{1}{2(n-1)}\left(g_{i j} \nabla_{k} R-g_{i k} \nabla_{j} R\right)\right],
$$

where $\mathscr{C}$ is the conformal curvature tensor [13].

Therefore, using $R_{i j}=0$ and $R=0$, equation (67) yields $\nabla_{h} \mathscr{C}_{i j k}^{h}=0$, that is, $\operatorname{div} \mathscr{C}=0$. In [18], Mantica et al. proved that an $n$-dimensional GRW spacetime satisfies $\operatorname{div} \mathscr{C}=0$ if and only if the spacetime is perfect fluid. Therefore, we conclude the following.

Corollary 2. A pseudo-Ricci symmetric GRW spacetime is a perfect fluid spacetime provided $A_{i} \neq \varphi u_{i}$.

Since $R_{i j}=0$ and $R=0$, from the definition of the conformal curvature tensor, it follows that $\mathscr{C}_{i j k}^{h}=R_{i j k}^{h}$. Hence, semisymmetric and conformally semisymmetric manifolds are equivalent. Eriksson and Senovilla [19] considered the semisymmetric spacetime and proved that it is of Petrov types $D, N$, and $O$. Thus, we have the following.

Corollary 3. A conformally semisymmetric pseudo-Ricci symmetric GRW spacetime is of Petrov types $D, N$, and $O$.

\section{Data Availability}

No data were used to support this study.

\section{Conflicts of Interest}

The authors declare that they have no conflicts of interest.

\section{Acknowledgments}

This work was supported by the King Saud University, Deanship of Scientific Research, College of Science Research Center.

\section{References}

[1] M. C. Chaki, "On pseudo Ricci symmetric manifolds," Bulgarian Journal of Physics, vol. 15, pp. 526-531, 1988.

[2] L. Tamássy and T. Q. Binh, "On weak symmetries of Einstein and Sasakian manifolds," Tensor, New series, vol. 53, pp. 140-148, 1993.

[3] U. C. De and A. Gazi, "On pseudo Ricci symmetric manifolds," Analele Stiintifice ale Universitatii Al I Cuza din Iasi-Matematica, vol. LVIII, pp. 209-222, 2012.

[4] F. Ozen and S. Altay, "On weakly and pseudo-symmetric Riemannian spaces," Indian Journal of Pure and Applied Mathematics, vol. 33, pp. 1477-1488, 2002.

[5] M. C. Chaki and T. Kawaguchi, "On almost pseudo Ricci symmetric manifolds," Tensor, New series, vol. 68, pp. 10-14, 2007.

[6] H. B. Yılmaz and S. A. Uysal, "Compatibility of $\varphi$ (Ric)-vector fields on almost pseudo-Ricci symmetric manifolds," 
International Journal of Geometric Methods in Modern Physics, vol. 18, Article ID 2150128, 2021.

[7] A. Gray, "Einstein-like manifolds which are not Einstein," Geometriae Dedicata, vol. 7, pp. 259-280, 1978.

[8] K. Hoda, C. A. Manticab, S. Shenawyc, and N. Syiedc, "Gray's decomposition on doubly warped product manifolds and applications," Filomat, vol. 34, pp. 3767-3776, 2020.

[9] U. C. De, H. M. Abu-Donia, S. Shenawy, and A. A. Syied, "On generalized projective $P$-curvature tensor," Journal of Geometry and Physics, vol. 159, Article ID 103952, 2021.

[10] C. A. Mantica and L. G. Molinari, "Riemann compatible tensors," Colloquium Mathematicum, vol. 128, no. 2, pp. 197-210, 2012.

[11] C. A. Mantica and S. Shenawy, "Einstein-like warped product manifolds," International Journal of Geometric Methods in Modern Physics, vol. 14, Article ID 1750166, 2017.

[12] C. A. Mantica, L. G. Molinari, Y. J. Suh, and S. Shenawy, "Perfect-fluid, generalized robertson-walker space-times, and Gray's decomposition," Journal of Mathematical Physics, vol. 60, Article ID 052506, 2019.

[13] L. P. Eisenhart, "Riemannian geometry," Princeton Landmarks in Mathematics, Princeton University Press, Princeton, NJ, USA, 1997.

[14] B.-Y. Chen, "Totally umbilical submanifolds," Soochow Journal of Mathematics, vol. 5, pp. 9-37, 1979.

[15] H. M. Abu-Donia, S. Shenawy, and A. A. Syied, "The $W^{*}$-curvature tensor on relativistic space-times," Kyungpook Mathematical Journal, vol. 60, pp. 185-195, 2020.

[16] C. A. Mantica and L. G. Molinari, "On the Weyl and Ricci tensors of generalized robertson-walker space-times," Journal of Mathematical Physics, vol. 57, Article ID 102502, 2016.

[17] C. A. Mantica and L. G. Molinari, "Generalized robertson-walker spacetimes-a survey," International Journal of Geometric Methods in Modern Physics, vol. 14, Article ID $1730001,2017$.

[18] C. A. Mantica, U. C. De, Y. J. Suh, and L. G. Molinari, "Perfect fluid spacetimes with harmonic generalized curvature tensor," Osaka Journal of Mathematics, vol. 56, pp. 173-182, 2019.

[19] I. Eriksson and J. M. M. Senovilla, "Note on (conformally) semi-symmetric spacetimes," Classical and Quantum Gravity, vol. 27, Article ID 027001, 2010. 